\title{
Reasoning about River Basins: WaWO+ Revisited
}

\author{
L. Oliva-Felipe · I. Gómez-Sebastià . \\ M. Verdaguer · M. Sànchez-Marrè · M. \\ Poch • U. Cortés
}

Received: date / Accepted: date

\begin{abstract}
This paper characterizes part of an interdisciplinary research effort on Artificial Intelligence (AI) techniques and tools applied to Environmental Decision-Support Systems (EDSS). WaWO+ the ontology we present here, provides a set of concepts that are queried, advertised and used to support reasoning about and the management of urban water resources in complex scenarios as a River Basin. The goal of this research is to increase efficiency in Data and Knowledge interoperability and data integration among heterogeneous environmental data sources (e.g., software agents) using an explicit, machine understandable ontology to facilitate urban water resources management within a River Basin.
\end{abstract}

Keywords Ontologies · Urban Water Systems • Water Resources Management - River Basin Management Environmental Decision Support Systems · Knowledge Management · Agent-based Simulation

\section{Introduction}

In the last decade, environmental researchers and professionals have recognized a strong need to control the complex socio-ecological nature of river basins $(R B)$ to sustainably manage water resources that are under pressure [37],

Prof. Cortés is a member of the Sistema Nacional de Investigadores (SNI), Mexico.

M. Verdaguer · M. Poch

(LEQUIA)

Universitat de Girona (UdG).

Campus de Montivili, E-17071, Girona, Spain. E-mail: \{marta.verdaguer, manuel.poch\}@udg.edu

L. Oliva-Felipe · I. Gómez-Sebastià · M. Sànchez-Marrè · U. Cortés

Knowledge Engineering and Machine Learning Group (KEMLg).

Universitat Politècnica de Catalunya (UPC).

C/Jordi Girona 1-3, E-08034, Barcelona, Spain. E-mail: \{igomez, loliva, ia $\} @$ @s.upc.edu 
[41], [44]. Dealing with the complexity of a $R B$ requires analytical frameworks that can handle the multiple scales and dimensions of environmental problems as well as uncertainties in the evolution of social systems. Therefore, $R B \mathrm{~s}$ have been represented using different analytical lenses, as follows: hydrological, ecological, institutional, etc. [17]. This is particularly true with urban water systems (UWS) where all of these elements acquire increasing important roles with the growth in human populations living there. From the AI point of view, this necessitates the design of an ontology that can support decision making, planning and reasoning. As noted by Medema [45] knowledge production and use in $R B$ and UWS management should accomplish the following:

- be coordinated across water and land resources;

- involve multiple stakeholders (those responsible for and affected by management interventions);

- support spatial and temporal scale integration;

- integrate disciplinary perspectives;

- enable to reason about environmental legislative and policy issues; and

- be holistic in character.

In these systems environmental data come from different sources, which are likely to be syntactically and semantically heterogeneous. Syntactic heterogeneity involves different data structure or formats [39] while semantic heterogeneity is defined as the differences in the objects and attributes that define the data, leading to disagreement on the meaning, interpretation and use of the same data [57].

Several initiatives are attempting to tackle environmental problems by offering structured vocabularies for biomes (ecosystem types), environmental features and environmental materials. Those vocabularies serve as the source of names and synonyms for identification processes (e.g. [67], [39], [32]).

In addition, the fast growing number of sensors connected to the Internet of Things (IoT) [61] is increasing the levels and capacities of monitoring. It is already producing large amounts of data that needs to be dealt with properly for various modelling, reasoning and decision making applications. A comprehensive state-of-the-art review of environmental knowledge-based modelling approaches can be found in [62].

\subsection{Ontologies and River Basins. The case of Urban Water Systems}

A context ontology aware is a shared knowledge representation and a reasoning mechanism used in AI to define the categories of things that exist within an application domain [13]. By contrast, domain information not included in the ontology cannot be reasoned about. Categories within the ontology represent the so-called designer's ontological commitments.

This commitment to a common ontology guarantees consistency but not completeness [52]. Ontologies establish a conceptually concise basis for communicating knowledge across a large community, and have already been applied to modelling environmental scenarios (see for example [23], [40], [63]. 
- Ontologies are viewed as the most advanced knowledge representation model;

- Ontologies support inference; this allows new derived knowledge to be generated from existing knowledge;

- Ontologies provide reasoning capabilities over existing knowledge through various user axioms/defined rules.

Domain knowledge for $R B$ s consists of three major parts, as follows: interpretation of data collected by sensors, regulation data published by government, and domain ontologies generated by researchers and professionals. Here, we will address all of them as $\mathrm{WaWO}+$ is able to represent all them (see $\S 4$ ).

Developing an ontology of a particular domain is not a goal in itself [50]. In this paper we present an ontology to support reasoning to make decisions related to the environmental management of water of the UWSs in a $R B$. In specific, we aim to derive facts that are not explicitly represented in the ontology or in the knowledge base and that help inform decisions. Anthropogenic uses of water, in agriculture, industry and domestic/urban contexts are an appropriate area for the application of ontologies, because the management of complex data, information and knowledge is a fundamental and growing concern. $R B$ management, to be efficiently applied in everyday practice, require that information is available, as in any other knowledge intensive activity.

An ontology for this purpose ought to support the following reasoning:

- Environmental elements that may suffer impacts and the type of impacts that may be provoked by natural or human actions.

- Stakeholder activities that produce these impacts, as follows: water use and reuse, potable and wastewater treatment, collection and distribution, optimal use, etc.

- Environmental indicators. Water quality indicators are used to measure the impact of actions and norms regarding water use.

- The social structure and how the relationships between the different stakeholders affect social goals.

The following physical elements should be considered during the reasoning process we have:

- Water: Service or product with a defined flow and quality requirement. The former is usually fixed by the local authorities by law. Representing those laws is also a key element.

- Infrastructure in an urban sector or city. In addition, geographical information is needed to locate each of those elements (see [53]). It encompasses components such as tanks, pumps, pipes or valves.

- Monitoring and control systems: Hardware (sensors, actuators) and software components (e.g. those that extract data from the physical system).

- End point users with service requirements.

As indicated by Mikulecký et al [46] this knowledge, as it happens with experts everywhere, may not be available when necessary for many reasons, as follows: 
- Experts do not need to be always available when necessary,

- Experts can suffer from common human problems, or suddenly their knowledge can be lost because of their mortality, or retirement,

- Experts can differ in their opinions on how to solve a particular situation.

The introduction of an ontological component in a UWS allows the development of tools which will contribute to the improvement of the current stateof-the-art, as follows:

- more stable operation through an ontology-based supervision;

- portability of the management system [8].

\subsection{Related work}

Ontologies play an important role in different aspects of EDSS development [38]. As noted by Parekh [51] the effectiveness and efficiency of environmental data usage greatly depends on the domain-specific understanding and empirical experience of the users in various application domains, such hydrology, environmental engineering, legal, etc.. The complexity and diversity of domain knowledge and terminology is one of the key obstacles for successful interdisciplinary studies. There is a vital need for an efficient mechanism for discovery and uniform representation and integration of relevant datasets.

Ontologies can be used to efficiently search in the domain as noted by Chau [25]. The assumption in almost all multi-tier component architectures and database search and access technologies is that in order for a client, being a human or a software component, to access a remote service (data or process), that client should know the semantics of the offered services [53]. Ontologies are a means to minimize semantic heterogeneity and simplify knowledge access and interchange [6]. The resulting semantic model should be usable, efficient, robust and authoritative.

Ontologies provide shared domain models that are understandable to both humans and machines. For water field, ontologies ([23], [22] and [7]) are usually the first step towards effective knowledge intensive tasks as diagnostic or assessment. They are a key element to management and model validation.

Garrido and Requena studied the case of Environmental Impact Assessment (EIA) that analyses the effects of human activity, ecosystem integrity and the quality of the environmental services that can be provided by them [35]. Similarly, Muñoz et al built an ontology where the environmental domain is represented based on the life-cycle assessment (LCA) methodology standardized in the ISO 1404X series (ISO14001) for setting an Environmental Management System (EMS) [47].

Moreover, several authors proposed ontologies for specific representation issues in $R B$ scenarios such as [43], who presented a generic ontology for conceptual design of treatment of flow sheets, [46] pointed out the need of an IoT approach to deal with the problem, [18] studied an activated sludge facility and a stabilization lagoon, [60] approached on the instrumentation and control of the treatment plants, [64] focused on different aspects of the Upper 
Ems River Basin, and [9] developed an argumentation-based framework for managing industrial wastewater discharges.

\subsection{Plan of the work}

The work in this paper is organized as follows: in $\S 2$ we describe target domain of this study going from a general definition to a specific $R B$, located in Catalonia, where the modelling takes place (see $\S 2.1$ ). We devoted $\S 3$ to revisit the original WaWO ontology, providing its main characteristics and describing its original reasoning capabilities [23]. In $\S 4$ we justify and discuss the extensions made to $W a W O$ to evolve it into $W a W O+$. In $\S 4.4$, we address the $W a W O+$ reasoning capabilities.

In $\S 5$, we describe the Agent-Based Simulator (ABS) we are using to model and reason about a $R B$ using $W a W O+$. This is a general purpose ABS. We have been trying to model the Besòs River Basin. In $\S 7$, we give our conclusions and discuss some hints about future work.

\section{The River Basin}

Many countries are placing growing demands on their rivers, such as using them to generate power, and for industrial cooling, impacting humans and wildlife. High population densities and transport networks put pressure on the water environment. Discharges from sewage works can impact on the quality of water or the enjoyment of it. In addition, the way land is managed has given rise to complex pollution and flooding issues. Diffuse pollution is a major pressure on the water environment and can come from urban areas as well as rural areas [27]. Managing water resources effectively requires a good information system for an appropriate decision-making activity.

Management of water resources in $R B \mathrm{~s}$ is hampered by a variety of uncertainties, such as unpredictability, incomplete knowledge and ambiguities, affecting problem identification and its solution. Uncertainty due to multiple knowledge perspectives arises because there are many actors with very different objectives and goals.

It is clear that several parameters influence each other and that various factors should be looked at when judging the water quality. We need to consider physical, chemical and biological parameters, such as:

- Physical: temperature, turbidity, conductivity.

- Chemical: Dissolved Oxygen, Biochemical Oxygen Demand or Chemical Oxygen Demand, hardness, $p H$, alkalinity, nutrients $(N$ and $P)$, toxic compounds.

- Biological/Ecological: biocenosis of bacteria, plants and animals, coliform bacteria and variety and complexity of the food chain. 
The combination of the criteria outlined above leads to river classifications, including very good, good, mediocre, deficient and bad ecological quality (a combination of physical, chemical and biological parameters)[20][31][19][30].

\subsection{The Besòs Basin}

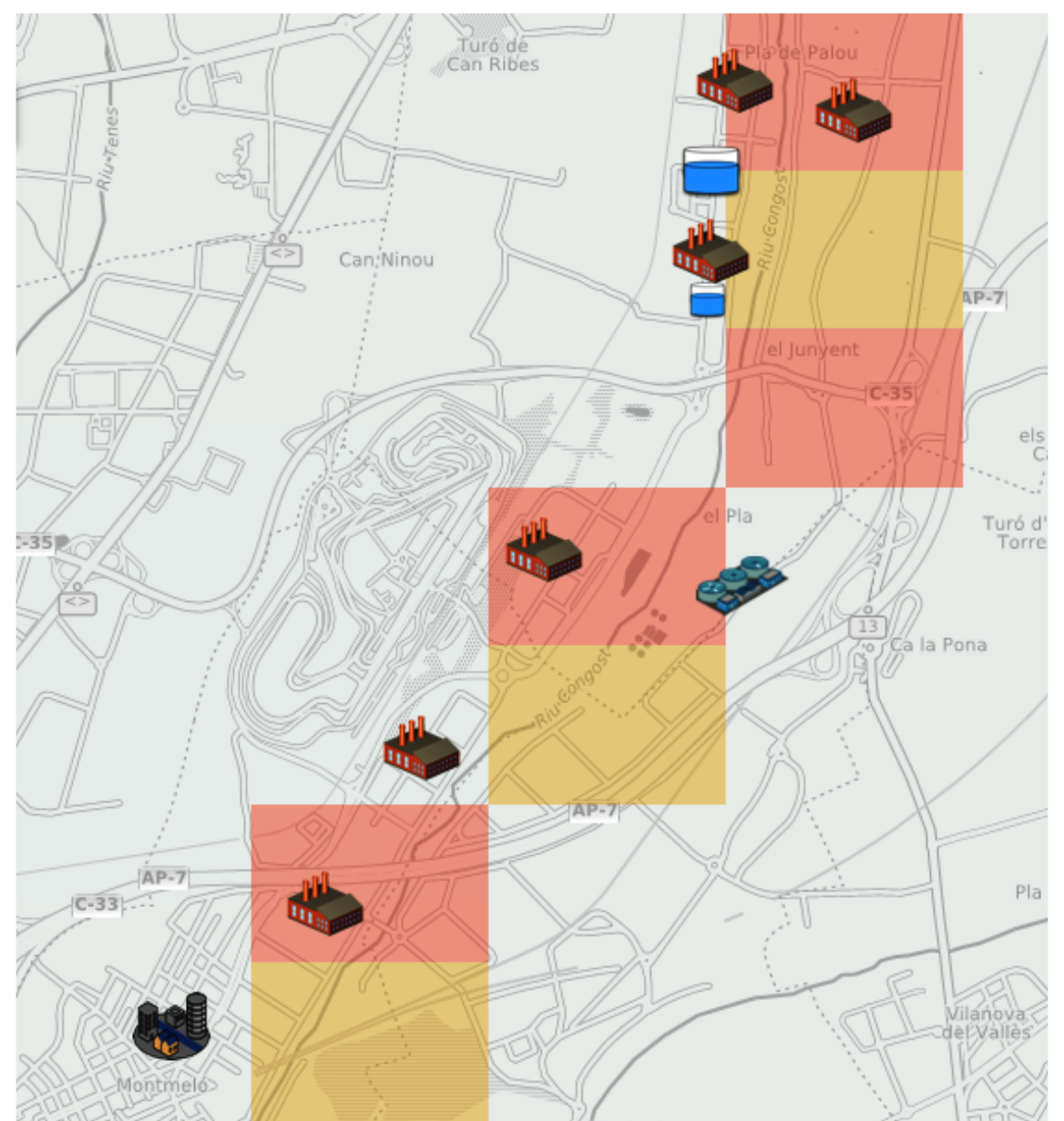

Fig. 1: The river basin scenario where $\mathrm{WaWO}+$ is applied (the Congost is an affluent of Besòs river)

The Besòs catchment $\left(1020 \mathrm{~km}^{2}\right)$ is located in Barcelona area and is a typical example of Mediterranean complex catchments. It is quite heterogeneous, including mountains over $1000 \mathrm{~m}$ tall to rural plains that have been 
suffering a continuous urbanization process over the last few decades. Rainfall is irregularly distributed throughout the year, with maximum rainfall in the fall (e.g. the flow in the river mouth during fall storms can be 100 times as strong as during the dry period, $2 \mathrm{~m}^{3} / \mathrm{s}$ ). The driest periods in the summer are in the flattest area near the sea and in the mountainous areas in the winter. The catchment area is one of the most populated catchments in Catalonia, containing more than $2 \mathrm{M}$ people. The working population is mainly occupied in industrial activities and in the sector of services. The type of industries installed in the catchment is varied with an important presence of the chemical, metallurgical, plastics, textile, building materials, paper, and food sectors. Overall, the catchment includes 10000 potentially polluting activities [2].

We used this catchment to model a real environment (see Figure 1) and to show that the proposed ontology $\mathrm{WaWO}+$ can cover most aspects that are needed when managing available water resources (see §4).

In previous research, we have focused on the final reaches of the Congost River, which is $70 \mathrm{~km}^{2}$ from where the river sustains the discharges of four towns connected to two wastewater treatment plants (WWTPs). In these studies, the elements under consideration were blocks of the UWSs as follows: the sewer system, the WWTPs and, the river itself [8], [29], [54]. Here, we are targeting a more ambitious goal.

\section{Ontologies}

By 2001, there was some terminological confusion among experts acting in a UWS, including: biologists, lawyers, economists, chemical and civil engineers, and plants managers. Each one used a particular vocabulary, and therefore, a precise common terminology was needed. To model that knowledge, an ontology was thought to be the best knowledge representation scheme. In an ontology, the concepts, the particular instances of a concept, their relations, as well as some constraining axioms regarding those concepts can be expressed. Usually, taxonomic relations ( $i s-a, s u b-$ part of ) are organized in a hierarchical way and constitute the main core of an ontology. In addition, the knowledge represented in an ontology is easily reusable (e.g., by exporting it into another ontology), and inferential reasoning processes can be run over an ontology, such as answering a query related to the ontological knowledge represented.

\subsection{WaWo}

Back in 2000, the $W a W O$ ontology was an attempt to build a model defining the meaning of each term/concept used in the wastewater domain, in a precise and unambiguous manner [21]. The ontology linked concepts/classes of individuals and instances/individuals at least with taxonomic/hierarchical relations and provided a terminology that each agent involved could understand 
and use. The basic categories/concepts in the WaWO model were represented as objects with specific properties and relations. Objects were structured into a taxonomy and the definitions of those objects, slots and relations were specified according to the Ontolingua [33] version of the Frame ontology. Objects included classes referring to the general wastewater domain, for decision support, as well as detailed micro-organisms taxonomy. Therefore, WaWO was represented in a frame-based formalism and stored at the Ontolingua Knowledge System Laboratory (KSL) Server of Stanford University. By October 2001, WaWO class hierarchy had 300 defined classes.

The WaWO ontology was defined and used to improve the supervision and management of a wastewater treatment plant. It was designed both to model the processes in WWTP and to enhance the reasoning process within the DAIDEPUR system execution [55]. The hierarchical organization of categories in $W a W O$ was expressed in the Ontolingua knowledge-representation language, and Knowledge Interchange Format (KIF) [36] axioms were used to answer queries, language analysis and general reasoning.

A reasoning mechanism provided by $W a W O$ was the explanation ability for the results of the Rule-Based Reasoning System (RBRS) [16] or the CaseBased Reasoning System (CBRS) [4]. The impasse resolution reasoning was a specific reasoning mechanism to enhance the performance of the OntoWEDSS system. When an impasse situation was reached, such as when the diagnosis output of the RBRS and the CBRS components were contradictory or when no diagnosis output at all was obtained, the $W a W O$ reasoning mechanism was activated to obtain a coherent diagnosis, or after using additional microbiological information, not integrated in the RBRS and the CBRS components, to obtain a successful diagnosis solution. This reasoning mechanism is inherited by $\mathrm{WaWO}+$.

The introduction of an agreed-upon ontology in the domain of wastewater biological treatment facilitated:

1. An accurate, effective communication and sharing of meanings, which in turn leads to other benefits such as knowledge reuse;

2. Advancing in the environmental technologies for the management of biological and biochemical processes;

3. Enhancing the knowledge about the specific microbial ecology of environmental processes developed in the technological ecosystems of treatment plants;

4. Improving the reasoning mechanisms to cope with impasse resolution situations.

\section{$4 \mathrm{WaWO}+$}

An important advantage of ontology-based systems can be found in an increased interoperability and reusability by using a semantic reference system [6]. To capture the knowledge necessary to reason and solving problems in a $R B$ we decided to expand the original $W a W O$ ontology [23] that was focused 
on reasoning about a single WWTP- and update the technology. In addition, $\mathrm{WaWO}+$ was designed to think about supplying the following aspects:

1. It offers unambiguous communication of domain concepts among agents that perceive and interact in a UWS or in a $R B$ scenario. That is a simple way to describe the key entities of the model and yet to infer many facts (conciseness);

2. It allows the inference of new knowledge from existing facts, allowing agents to have a more accurate information of their environment;

3. It is authoritative for domain experts;

4. Move from real, grounded concepts to more abstract ones, thus effectively enabling agents to have a higher-level, domain-free reasoning capability;

5. It can be combined with other ontologies that represent dynamics occurring when consuming resources from the river (e.g., using as part of a wastewater system).

WaWO was inspired in METHONTOLOGY [34] and implemented in Frames. While $\mathrm{WaWO}+$ was based in our experience in building ontologies and we decided as formalization tool we use Protégé [49] in $O W L$ format [42]. The $O W L$ Web Ontology Language is a modelling tool aimed to describe ontologies and represent knowledge using formal semantics encoded in $R D F / X M L$ formats, thus allowing to be consumed by other computer entities that may reason about the domain to achieve their goals. Protégé supports the tradeoff between interoperability and usability of knowledge-based systems. These properties allow $\mathrm{WaWO}+$ to import other ontologies, committing to all of their classes, properties and constraints, and experts supported the decision of which ontologies and classes need to be included. Protégé supports Web 2.0 technologies and applications. The internet has become the primary environment in which people and machines communicate, exchange content, and collaborate to solve problems, and $\mathrm{WaWO}+$ is meant to be web accessible.

As a strategy for identifying concepts domain experts were consulted to expand $\mathrm{WaWO}$ towards $\mathrm{WaWO}+$ and a substantial set of taxonomic concepts were added as a result of this. In the first stage, experts revisited WaWO to update it and identify possible extensions. Three were selected: a) Water Quality, b) Social aspects, and c) Urban water cycle (see Figure 2). Normative regulations were also analysed to extract terms and definitions used to regulate the water management domain. Work performed by [24] was also used to aggregate geographical features that may be present in a $R B$ (e.g., lakes, stream, lagoon). The actual $\mathrm{WaWO}+$ is the evolution of several prototypes, in those prototypes a sizeable part of the ontology is very detailed; the remainder is specified in deeper detail in the successive versions.

The following subsections introduce such extensions.

\subsection{Water quality extension}

The water quality extension of the WaWO ontology covers the evolution of wastewater treatment philosophy from when it was published until now. For 


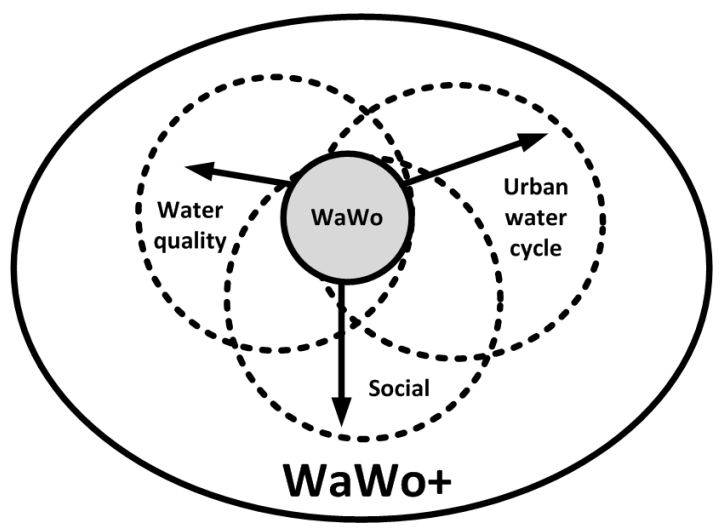

Fig. 2: The $W a W O+$ ontology extensions. Main additions to the original $\mathrm{WaWO}+$ ontology cover the urban water cycle, social and water quality aspects.

instance, the extension reflects a change in paradigm from pollutants to water quality measures. For example, the wastewater that an industry discharges to the sewage system has specific pollutant concentrations as a subproduct/result of its productive processes. These imply different water quality indicators, depending on the industrial sector the industry belongs to (e.g. a slaughterhouse usually discharges wastewater with a higher biological demand oxygen indicator and suspends solids concentration while paperworks produce higher chemical concentrations). WaWO+ enables water bodies to be defined according to their water quality indicators and permits to reason about them.

- Figure 3a shows the set of physical and chemical water indicators that can be used to characterize a water mass, by means of the concentrations of these indicators (see Figure 3b). Some of them were already defined in the original WaWO (e.g. SuspendedSolid, Conductivity or Turbidity). Figure $3 \mathrm{c}$ shows new indicators that also expand to those already in $W a W O$ such as EmergingContaminants (e.g., antibiotics, beta blockers, analgesics) and HeavyMetals. WaWO+ not only expands the set of indicators but classifies them differently to reflect the change in perspective mentioned above. While WaWO strongly focused on WWTP internal aspects, $W a W O+$ adds more fine-grained concepts (e.g., from the initial Metals concept to HeavyMetals and a set of subclasses that refer to specific heavy metals that affect water quality), so these quality measures can be applied to different types of water masses (see $\S 4.3)$.

Assessing water quality or reasoning about normative aspects requires determining the water quality of certain parts of the river. For instance, it is necessary to determine whether water close to urban areas can be considered to be clear water. 


\begin{tabular}{|c|c|}
\hline $\begin{array}{l}\text { Chemical } \\
\text { Chloride } \\
\text { Hardness } \\
\text { HydrogenIonConcentration } \\
\text { BiochemicalOxygen Demand } \\
\text { ChemicalOxygenDemand } \\
\text { SuspendedSolid } \\
\text { TotalNitrogen } \\
\text { Ammonia } \\
\text { Nitrate } \\
\text { Nitrite } \\
\text { OrganicNitrogen } \\
\text { TotalPhosphorus } \\
\text { OrganicPhosphorus } \\
\text { Ortophosphate } \\
\text { Poliphosphate }\end{array}$ & $\begin{array}{l}\text { Physical } \\
\text { Colour } \\
\text { Conductivity } \\
\text { Odour } \\
\text { Taste } \\
\text { Temperature } \\
\text { Turbidity }\end{array}$ \\
\hline
\end{tabular}

(a) Chemical and physical water indicators

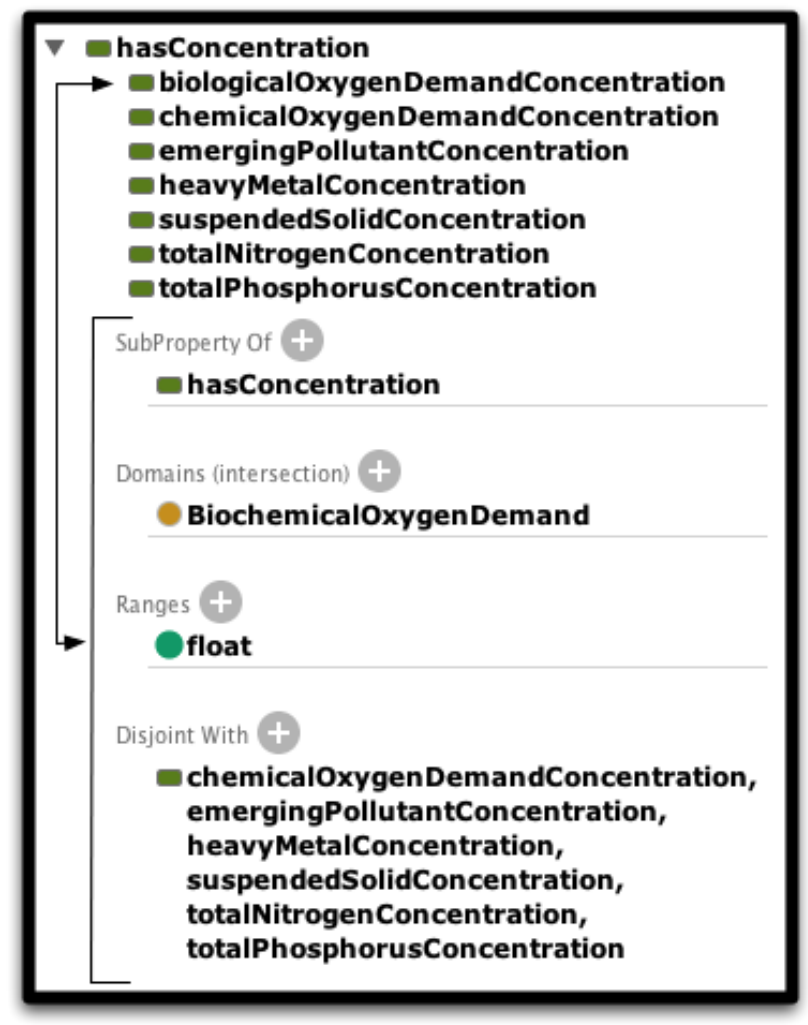

(b) Properties of water indicators

\begin{tabular}{|c|c|}
\hline $\begin{array}{l}\text { FmergingContaminant } \\
\text { AnalgesicAntiinflammatory } \\
\text { AcetylsalicylicAcid } \\
\text { Diclofenac } \\
\text { Ibuprofen } \\
\text { Naproxen } \\
\text { Antibiotics } \\
\text { Betablockers } \\
\text { CholesterolRegulators } \\
\text { PsychiatricUse } \\
\text { XRayContrastMedia }\end{array}$ & $\begin{array}{l}\text { FeavyMetal } \\
\text { Aluminium } \\
\text { Cadmium } \\
\text { Chromium } \\
\text { Copper } \\
\text { Lead } \\
\text { Mercury } \\
\text { Nickel } \\
\text { Zinc }\end{array}$ \\
\hline
\end{tabular}

(c) Emerging contaminant and heavy metal water indicators

Fig. 3: $W a W O+$ ontology for water quality indicators 


\section{$\nabla$ WaterMass \\ Flow_water_mass \\ Static_water_mass}

(a) Water bodies

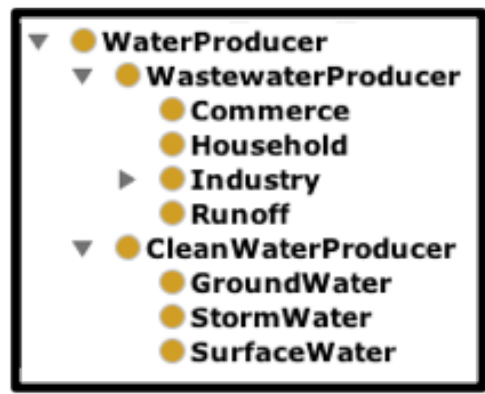

(c) Water sources

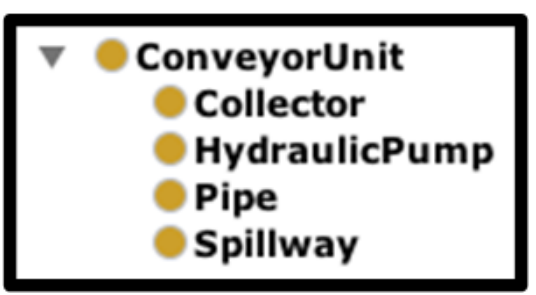

(b) Wastewater network

Fig. 4: Different elements in $\mathrm{Wa} W O+$ ontology.

- WaWO+ provides a classification of different types of water that distinguishes water bodies from a chemical perspective (e.g., clean water, rain, wastewater) and its location or dynamism (e.g., flowing/standing water, ground/surface water). Figure 4 a shows the extension of $\mathrm{WaWO}+$ performed to classify water bodies;

\subsection{Urban water cycle extension}

WaWO focused on the processes and operational concepts of WWTP. The urban water cycle extension includes a more integrated approach with the objective of better embracing the urban water scenario. This extension includes the following concepts:

- Processes, including water treatment operations performed in the different elements of urban water cycle both for drinking water and wastewater treatment.

- Network system for water distribution and sewage systems, which includes additional elements such as water retention tanks and pipe infrastructure.

Figure $4 \mathrm{~b}$ depicts the elements of both potable and wastewater networks. Those include conveyors, pipes, spillways, pumps and tanks. 
Figure 4c depicts the different water sources, including natural clean water sources and wastewater producers. The sources of clean water are classified following the current legislation according to their origin. The wastewater producers follow the type of generators (producing domestic, industrial and meteorological wastewater)[31][19][30]. In our scenario, we distinguished between headwaters that continuously feed the river according to seasonal water regimes. We also distinguished between at least two different wastewater producers, including industries and households (e.g., cities and towns). Finally, we also considered weather phenomena, such as rain, which produces large masses of water that are collected through the sewage system as well as run-off water that are directly received in the river, see figure 8 .

A sewage network is composed essentially of pipes that connect industries and households to transport their wastewater to a WWTP and, after proper treatment, to the river or sea. Several other components can also be found, including conveyors, which connect smaller sewage networks into a general one, collectors to laminate wastewater influents and exercise some control over the wastewater flows on the network or storage tanks to retain sudden heavy rainfalls (meteorological retainers) to avoid overfilling the network (which is a common phenomenon in Mediterranean weather). A WWTP needs to incorporate this knowledge during its reasoning processes (see $\$ 4.4$ ), or even when negotiating with wastewater produces when they can perform a discharge or how much time it takes the water to flow until its destination.

\subsection{Social extension}

By including actors that interact with integrated urban water cycle in $\mathrm{WaWO}+$ we are effectively creating a society of agents interacting in the river basin scenario. This society includes actors that interact between each other to achieve both individual goals, which are particular to each actor, and common goals, which are shared by the whole society of agents. To ensure that the particular goals are aligned with common ones and facilitate interactions between the different actors, a normative structure is introduced to define the expected patterns of behaviour. The extension includes the following concepts:

- Norms and regulations, including European Water Framework Directive (WFD), Spanish and Catalan regulative bodies, as well as other normative concepts. This extension allows the actors involved in the scenario to reason about the normative framework, effectively providing norm-awareness.

For instance, the European Council directive for wastewater treatment [19] in Article 4 and the Catalan plan for wastewater treatment inspired on this directive [28] state:

Member States shall ensure that urban wastewater entering collecting systems shall before discharge be subject to secondary treatment or an equivalent treatment as follows: 
Norm $N_{1}$ : Let $W_{i} \in \mathcal{W}$ be a WWTP, $M_{j} \in \mathcal{M}$ a water mass and $T_{k} \in \mathcal{T}$ a secondary treatment. Once $W_{i}$ receives a particular water mass $M_{j}$, the plant has the obligation to treat the water mass with secondary treatment $T_{k}$ before discharging the water mass. Sanction $S_{1}$ : A generic sanction is applied to the WWTP if the norm is not complied with.

\begin{tabular}{|c|l|}
\hline Activation Condition $N_{1}$ & received $\left(W_{i}, M_{j}\right)$ \\
\hline Expiration Condition $N_{1}$ & discharged $\left(W_{i}, M_{j}\right)$ \\
\hline Maintenance Condition $N_{1}$ & True \\
\hline Deadline $N_{1}$ & $\begin{array}{l}\text { performed }\left(T_{k}, W_{i}, M_{j}\right) \wedge \\
\text { counts_as }\left(T_{k}, \text { SecondaryTreatment }\right)\end{array}$ \\
\hline Activation Condition $S_{1}$ & isViolated $\left(N_{1}, W_{i}\right)$ \\
\hline Expiration Condition $S_{1}$ & GenericSanction $\left(W_{i}\right)$ \\
\hline Maintenance Condition $S_{1}$ & True \\
\hline Deadline $S_{1}$ & True \\
\hline
\end{tabular}

Fig. 5: Example of formal norm specification for obligation

\begin{tabular}{|c|c|c|}
\hline $\begin{array}{l}\nabla \text { NormComponent } \\
\text { ConstitutiveComponent } \\
\text { Context } \\
\text { InstitutionalFact } \\
\text { BruteFact } \\
\text { DeonticComponent } \\
\text { MainteinanceCondition } \\
\text { ExpirationCondition } \\
\text { ActivationCondition } \\
\text { Deadline } \\
\text { RepairCondition }\end{array}$ & $\begin{array}{l}\text { Variable } \\
\nabla \text { Norm } \\
\nabla \text { RegulativeNorm } \\
\nabla \text { DeonticNorm } \\
\text { Obligation } \\
\text { Permission } \\
\text { Prohibition } \\
\text { Sanction } \\
\text { ConstitutiveNorm } \\
\text { Function }\end{array}$ & $\begin{array}{l}\text { Operator } \\
\text { Quantifier } \\
\text { Exists } \\
\text { ForAll } \\
\text { Implication } \\
\text { And } \\
\text { Not } \\
\text { Or } \\
\text { Formula } \\
\text { Constant }\end{array}$ \\
\hline
\end{tabular}

Fig. 6: Normative model in $\mathrm{WaWO}+$

- At the latest by 31 December 2000 for all discharges from agglomerations of more than 15000 p.e. (population equivalent)

- at the latest by 31 December 2005 for all discharges from agglomerations of between 10000 and 15000 p.e.

This means that by '01 January 2006', all WWTPs $W W T P_{i} \in \mathcal{W}$ with a p.e. of 10.000 or more have the obligation to perform a secondary treatment (or a treatment that counts-as secondary treatment, that is, an equivalent) before discharging water to the river. Failure to comply with the norm will result in the WWTP being sanctioned. Figure 5 shows the formal specification of the regulative norm in our model.

Figure 6 depicts the extension of $\mathrm{WaWO}+$ performed to support our formal norm specification. The specification is based on the model presented in [5]. Norm components are formed by a formula. A formula is a set of functions with constants and variables connected by operators. Norms can be constitutive or regulative [14]. Constitutive norms are formed by brute facts, institutional facts and a context. Regulative norms contain activation, maintenance, expiration, deadline and repair conditions. Deontic norms represent the obligations, 
prohibitions and permissions in the normative system. Repair conditions are captured by sanctions that activate when deontic norms are violated.

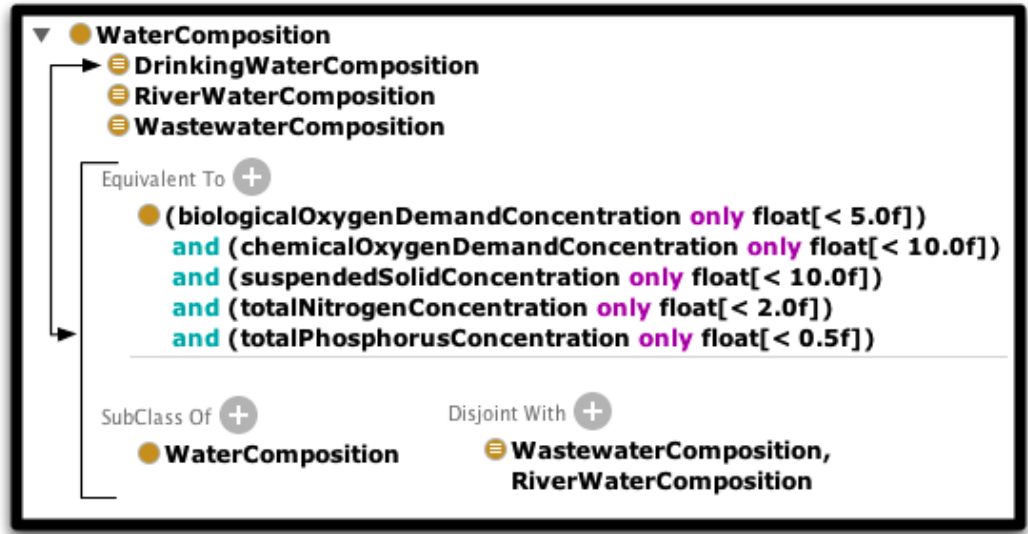

Fig. 7: Social water classifications in $W a W O+$ and the rules to classify according to the composition of water masses

Figure 7 shows water classification from a social point of view, including wastewater (i.e. polluted water to be treated), river water (i.e. clean water with bad taste or odour parameters) and drinking water (i.e. clean water for human consumption). It is also shown the rules that define the class expression and allows the reasoner to classify instances of water masses accordingly. This allows to introduce new water masses along the sensor readings that measure the water indicators (see Figure 3) and infer its classification according to legislation limits.

\subsection{Reasoning with $W a W O+$}

As described in $\S 3.1, W a W O+$ inherits $W a W O$ reasoning mechanisms to cope with impasse resolution situations (i.e. when no diagnostic is provided by the EDSS) [23]. The original idea was to connect a CBR and an EDSS with WaWO to support the CBR cycle with inferences from it.

Now, WaWO+ is written in OWL and is therefore equipped with unambiguous semantics. This allows devising reasoning algorithms that enable the discovery of knowledge that is only implicit in the model. Agnostic-domain reasoners, such as Pellet [59], can be used to exploit it. Furthermore, agents using the ontology can effectively use the normative structure introduced in $\mathrm{WaWO}+$ as a basis for more complex reasoning mechanisms based on deontic logics [15] and inference of the social implications of events in the world [56] as depicted on the following example. 


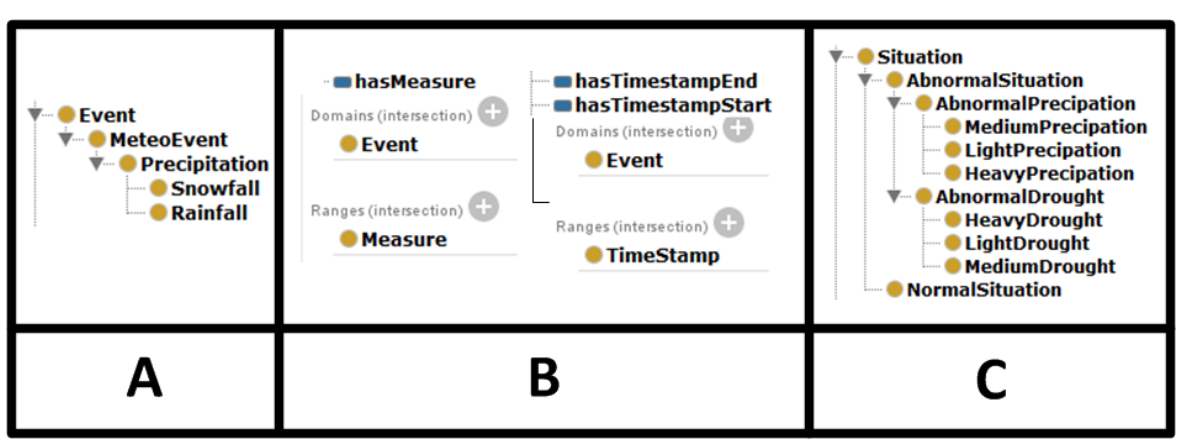

Fig. 8: Meteorological data and situations in $\mathrm{WaWO}+$

Figure 8 depicts the different elements used to socially interpret meteorological situations. Figure $8 A$ depicts the meteorological events [1] supported in the current version of $\mathrm{WaWO}+$, mainly Precipitations in the form of Rainfall and Snowfall. Figure $8 B$ shows the main properties of meteorological events, mainly a starting and ending time and the amount of precipitation (in the form of cubic metres) accumulated during the duration of the event. The class Time-Entity is included in $\mathrm{WaWO}$, and in principle this allows temporal reasoning. It is important to note, cases such as Precipitation that have high variability; in this case, in terms of cubic metres/minute (e.g. covering both periods of heavy and light rain), the event can effectively be divided into events with shorter duration. Finally, Figure $8 C$ depicts the classification of meteorological situations. $W a W O+$ is able to cover periods of both rain and absence of rain (i.e. Drought) in the form of severe (heavy), medium and light situations. The classification is performed by agents using $\mathrm{WaWO}+$ to reason about the history of events, effectively classifying the actual situation as normal or abnormal.

It is important to notice that, HeavyRain and HeavyDrought situations are not exclusive. We can find a situation where, after a period of HeavyDrought (i.e. more than 90 days without significant Precipitations), a huge quantity of rainfall during a very brief interval of time. This type of Precipitation is very common in the Mediterranean area, and due to their brief duration, the water cannot be stored or used effectively. Therefore, these types of events are considered rain but they simultaneously extend drought situations.

The normative structure present in $W a W O+$ (as introduced later in §5) defines the classification rules that allow the interpretation of meteorological events (or the absence of them), as one of the available situations. This classification is tightly coupled to the geographical region in which $\mathrm{WaWO}+$ operates because heavy rains, droughts or snowfalls are not considered equally in continental and Mediterranean climates. Figure 9 shows an example of a classification rule for heavy rains in the Mediterranean area. Please note, a similar classification rule can be used for the classification of Clean Water 


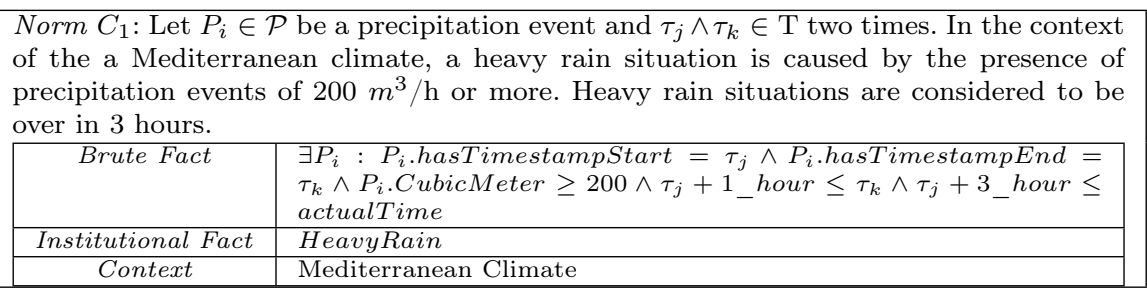

Fig. 9: Example of constitutive norm for heavy rain situations

Producers and Wastewater Producers, as observed on Figure 4c. In this case, the Brute Fact analyses a water mass in terms of water quantity and quality (i.e. pollutant concentration) effectively classifying it as an available water source via the Institutional Fact.

\section{Multi-Agent System Simulation}

In the following section, we will outline the general idea of simulations -as well as the specific concept of Multi-Agent System (MAS) simulations- and explain which features make them particularly suitable for studying a $R B$. We propose a bottom-up approach to create a model that simulates the interactions of different actors in a $R B$. The result of a simulation is the representation of the behaviour or key characteristics of a physical or abstract system through the use of another system.

The simulation system is an abstract and simplified computational representation of the real system to analyse a specific problem of the real system (e.g. the effect of enforcement mechanisms or the emergence of behavioural patterns). By abstracting from the real system, simulations reduce the complexity of the problem being analysed and allow for problem-oriented experimentation, which might otherwise not be possible in the real system [12], likes the Besòs basin.

A MAS is the representation of a system as a collection of autonomous selfdirected decision-making entities, so-called agents. An agent is a computer system that is situated in some environment and is capable of autonomous action in his environment to meet its delegated objectives [66]. As defined by Tube [12], these agents engage in complex, often non-linear interactions. They act locally and individually towards their own goal, based on their assessment of their current situation with the help of a basic set of agent-individual rules.

The MAS can model a society in which the agents coordinate and interact with the environment as well as among them. The agents' behaviours are driven by a set of norms (policies) and the goals they intend to achieve (e.g. ensuring a certain level of water quality in a river). In our specific case, we have opted to describe such norms with deontic specifications. Deontic logic allows describing a set of obligations and permissions that are intended to 
Norm $N_{3}$ : Let $W W T P_{i}$ be a Waste Water Treatment Plant, $W_{j}$ a water mass and concentration $\left(W_{j}, H g\right)$ the concentration of mercury in the water mass. It is forbidden for Waste Water Treatment Plants to discharge water masses to the river with a mercury concentration higher than $0.005 \mathrm{mg} / \mathrm{l}$. Please note the norm is always active, as the prohibition always holds, therefore activation condition is set to True and expiration condition to False.

Sanction $S_{3}$ : A generic sanction is applied to the Waste Water Treatment Plant if the norm is not complied with.

\begin{tabular}{|c|l|}
\hline Activation Condition $N_{3}$ & True \\
\hline Expiration Condition $N_{3}$ & False \\
\hline Maintenance Condition $N_{3}$ & $\begin{array}{l}\text { discharged }\left(W W T P_{i}, W_{j}\right) \wedge \text { concentration }\left(W_{j}, H g\right) \quad \geq \\
0.005 m g / l\end{array}$ \\
\hline Deadline $N_{3}$ & \\
\hline Activation Condition $S_{3}$ & isViolated $\left(N_{3}, W W T P_{i}\right)$ \\
\hline Expiration Condition $S_{3}$ & GenericSanction $\left(W W T P_{i}\right)$ \\
\hline Maintenance Condition $S_{3}$ & True \\
\hline Deadline $S_{3}$ & True \\
\hline
\end{tabular}

Fig. 10: Example of formal norm specification for prohibition.

guide agents' actions effectively facilitating their decision-making processes. These obligations and permissions conform to the norms of our system and are a representation of real-world institutions that govern the environment in which the agents are limited. Figure 10 shows an example of a deontic prohibition based on European wastewater directives.

These agents can make use of ontologies to make a deeper reasoning during their decision making process, as follows: what they perceive in their environment, the implications of breaking a norm or the indirect effect of their actions. Enabling agents to make use of an ontology to allow them to manipulate domain-related concepts and infer new knowledge as well as using higher-level abstractions in their reasoning and communication with other agents. This facilitates the implementation of more complex models of agent behaviour.

\subsection{Our Multi-Agent System Simulation Architecture}

Our proposed system is a LISP-based platform with the following main elements (see Figure 11):

- agents, which all share a common structure based on a deliberative architecture. We distinguish between industries, WWTPs, wastewater producers, (e.g. industries, households, etc.) and the competent authority; the latter makes use of a monitor component which continuously reads the events occurring in the system to enforce enacted norms;

- an engine, who is responsible of executing (evaluating) the reasoning process of each agent, as well as the actions and messages derived from their plans;

- an environment, which models a $R B$; 


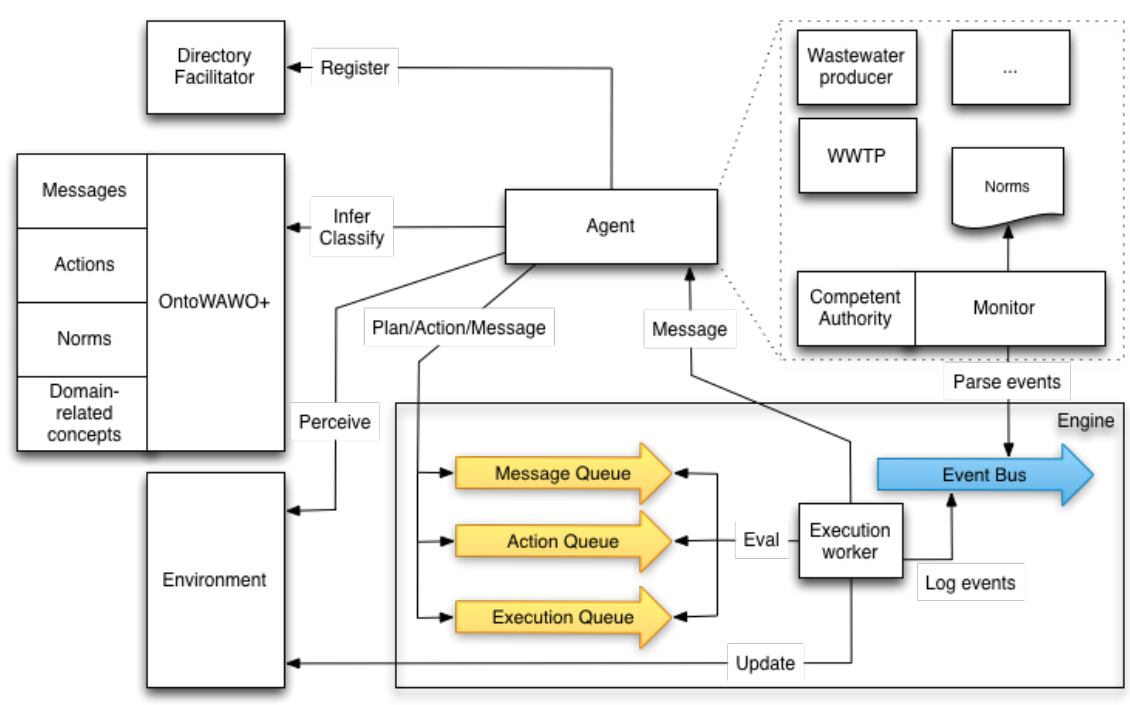

Fig. 11: Multi-Agent Simulation System architecture.

- a directory facilitator, which takes care of keeping a register of all the agents in the system. It also acts as yellow pages to locate a specific agent for interaction/messaging purposes. After registering in the platform, the agent is in the execution queue to start its reasoning;

- WaWO+ which contains several domain-related concepts of the modelled $R B$ scenario. It is used by agents to reason about their actions, update their beliefs with higher-level abstractions as well as to communicate with other agents in such a way that it is decoupled from ad hoc messages or raw data.

The agents (i.e. industries, WWTPs or the competent authority) are meant to use $\mathrm{WaWO}+$ during their reasoning and planning. This enables agents to perceive the environment and reason with a higher level of abstraction; for instance, perceiving the different pollutant concentrations as well as indicators and classifying the water as clean/polluted or acceptable/non-acceptable according to whatever the norms may impose on certain areas of the $R B$. Afterwards, they can reason according to those abstractions and send messages to other agents, so their current situation can be easily understood if necessary, instead of relying on what others may interpret. Furthermore, these classifications can be performed in accordance to current norms and regulations. Even if these norms change the interpretation of perceived facts (e.g. a water mass with a certain pollutant concentration as highly polluted when it was previously considered as fresh water) their reasoning and plans are decoupled but the resulting behaviour, messages and plans will change accordingly. 


\begin{tabular}{|c|c|c|}
\hline Feature & WaWO & WaWO+ \\
\hline Format & Frames & OWL/RDF \\
\hline Number of classes & 109 & 233 \\
\hline Number of instances & 0 & 0 \\
\hline Number of properties & 2 & 22 (object) / 18 (data-type) \\
\hline Number of class at 1, 2 and 3 level & $15,14,18$ & $27,63,89$ \\
\hline Number of class leaves & 85 & 176 \\
\hline Average depth & 4.7 & 3.12 \\
\hline Highest depth level & 6 & 6 \\
\hline
\end{tabular}

Table 1: WaWO and $W a W O+$ comparison

The monitor component is used to observe the agents' actions in the scenario, effectively detecting patterns of behaviour that are non-compliant with the society's expectations as defined in a set of common norms. Such set of norms requires a mutual understanding of terms as supported by $\mathrm{WaWO}+$. This allows domain experts to easily model common behavioural patterns and high level goals shared by the society of agents (e.g. balance water quality and economic outcome).

\section{Evaluation}

$W a W O+$ expands $W a W O$ not only in content but also in many other features. As explained in $\S 4$, the most prominent difference is in the programming language used. Instead of the original Frames language, $\mathrm{WaWO}+$ is built by means of OWL/RDF. This change allows the use of Description Logics which allows for certain, and desired, expressive features such as reasoning and inference [11]. This feature, in fact, improves $W a W O+$ 's usability with respect to $\mathrm{WaWO}$. In addition, reasoning capabilities were used during $\mathrm{WaWO}$ +'s development (e.g. to eliminate local inconsistencies). The more expressive the ontology language, the more precisely the intended meaning of a vocabulary can be specified, and consequently, the more precise conclusions can be drawn. For example, introducing disjointedness axioms greatly facilitates consistency checking and the automatic evaluation of individuals in a knowledge base with regards to a given ontology [65]. It also eases the task of consuming the ontology through the Web, which is one of the WaWO+'s (secondary) purposes.

When examining Table 1 , one can observe that structurally $\mathrm{WaWO}+$ has increased the expressiveness of the original $(\mathrm{WaWO}+$ has more than doubled the number of concepts and properties, particularly the number of classes at second and third level). The average depth has been reduced, as a result of the $W a W O$ refinement process for its generalization and expansion. $\mathrm{WaWO}+$ has also incorporated new properties thus moving from a hierarchical taxonomy provided in $W a W O$ to a full ontology. We then proceeded with an expert-based evaluation where different questions were asked towards to verify the ontology was capable to answer as well as to check if classification was correct according to their expertise. An example of query was the following one: 


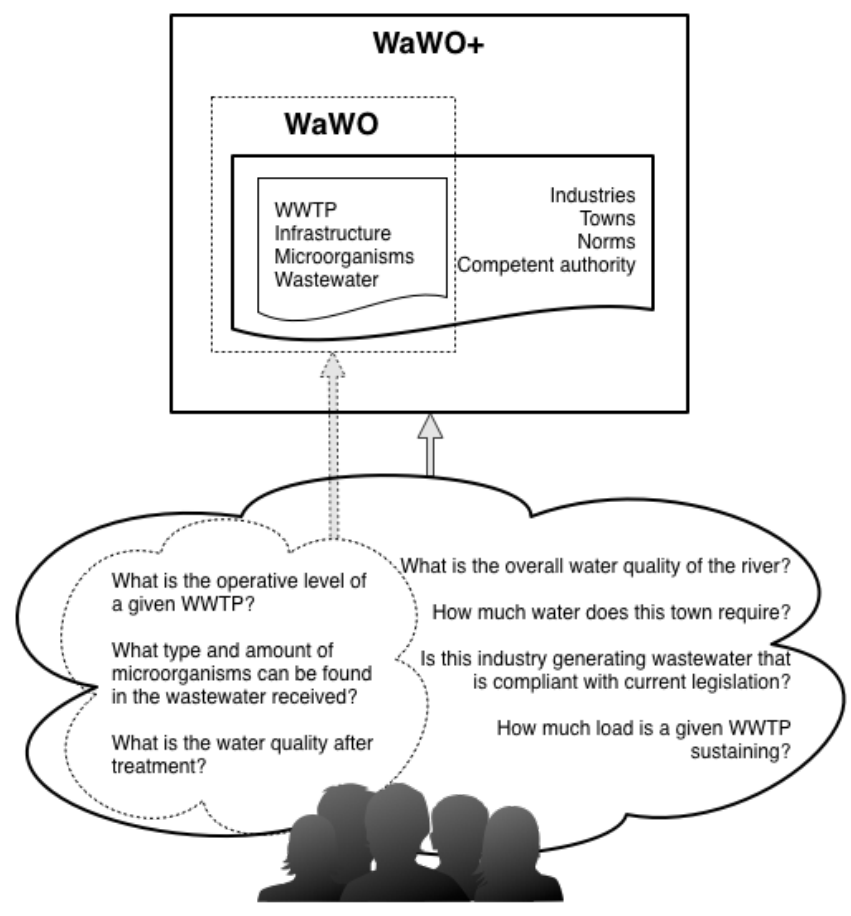

Fig. 12: $W a W O+$ expands $W a W O$, allowing more questions to be asked and answered. The right hand part of the boxes depict $\mathrm{WaWO}+$ 's actual extension.

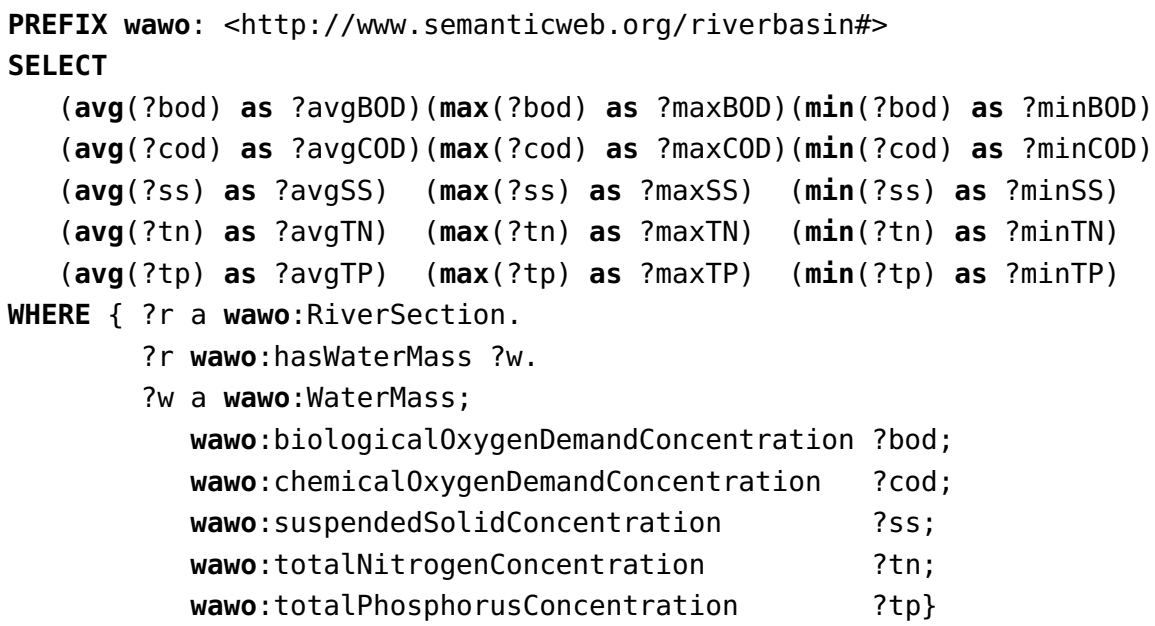

Listing 1: SPARQL query 


\begin{tabular}{|c|c|c|c|}
\hline Water Indicator & AVG & MAX & MIN \\
\hline$B O D$ & 20.34 & 24.03 & 18.76 \\
\hline$C O D$ & 21.36 & 22.15 & 19.05 \\
\hline$S S$ & 29.88 & 33.40 & 27.51 \\
\hline$T N$ & 8.42 & 8.54 & 8.11 \\
\hline$T P$ & 0.77 & 0.85 & 0.70 \\
\hline
\end{tabular}

Table 2: Results from executing query Listing 1

Afterwards, we created a fictitious water mass whose indicators where the average values we queried before; then, the water mass was inserted in the ontology. The resulting inferred classification determined that, in overall, the river quality was considered as RiverWaterComposition. However, the $\max / \min$ deviation from the average value suggested there were some river sections that might be not as clean as it seemed.

Therefore, $W a W O+$ has effectively expanded its predecessor, allowing the users to ask a wider range of questions (see Figure 12), and provides a broader model of the river basin domain.

\section{Conclusions and Future work}

This paper presents $\mathrm{Wa} W O+$ and its architecture and discusses the rationale that supports the extensions to the original $W a W O$ ontology. The WaWO+ was built because previous research in the field (e.g. [29], [8], [10], [48]) has encouraged us to use more sophisticated techniques in knowledge representation and management due to the number of actors, the large quantity of variables and the complexity of the different processes involved in reasoning about wastewater treatment UWS in $R B \mathrm{~s}$, in particular in the Besòs Basin.

The original $W a W O$ ontology was developed to represent knowledge about the micro-organisms involved in biological wastewater treatments. In turn, the extensions of $\mathrm{WaWO}+$ (see figure 4) allow for more complex forms of reasoning including the expansion to the scope of argumentation to normative aspects. It also includes a wide range of concepts related to environmental aspects on water management in the UWSs. As Vatn [3] identified, the understanding of what institutions are and do is the core issue. When reasoning about $R B$ issues, one must also acknowledge that communication, calculation, and choices, are not cost free. The idea of bounded rationality is a response to this problem [58]. In [10], we only addressed industrial water discharges.

Ontologies provide an abstract conceptualization of information to be represented and machine-interpretable definitions of basic concepts in a specific domain and the relations among them. $W a W O+$ is a step ahead in the advancement in reasoning about water management in $R B \mathrm{~s}$. This is critically dependent on the proper integration of water information.

The way we designed $W a W O+$ clearly improves $W a W O$ because it allows performing more complex reasoning tasks. WaWO+'s reasoning capability is 
regarded as a tool and service to help users that enables a direct query functionality and allows an advanced inference functionality. We foresee the use of $\mathrm{WaWO}+$-like ontologies in the development of River Basin Management Plan, as in [27]. We expect $W a W O+$ to grow and be enhanced with the integration of new services and their Ontological description.

Ontologies should be considered as infrastructures that enable knowledge sharing and reuse. This is a key factor to cope with the interaction of many actors with different goals and, possibly, competing interests. Building ontologies such as $W a W O+$ or $K M$ [25] are a crucial element when reasoning about complex problems in which heterogeneous agents intervene and where incomplete information and uncertainties are large. Problematic situations in a UWS might be complex, and agents may be needed to elucidate about the elements perceived, their relations and the exact meaning or, what implications may have their actions that should be unambiguously communicated.

$W a W O+$ users can be decision makers, experts, $R B$ authorities, stakeholders and citizens with different levels of access to the available information ${ }^{1}$. The OWL representation is web oriented and allows knowledge sharing through the Internet.

\subsection{Future Work}

In the IoT, new water management services will be deployed [46]. For example, storm water management and smart grid water will see growth. IoT services will bring the incorporation of stakeholder measurements, local knowledge, experience and beliefs of their own environment. This participation has to be properly addressed and the produced information included in the system. This implies capturing and leveraging the provenance data to increase its extensibility and transparency (see [65] for a complete discussion on these issues.). In addition, it is expected that WTTPs will evolve into bio-refineries. For example, new, innovative wastewater treatment processes will enable water recovery to help close the growing gap between water supply and demand.

$\mathrm{WaWO}+$ should evolve and be transformed from a contextual ontology into a context awareness ontology. Ontologies are key requirements for building pervasive context-aware systems, in which independently developed sensors, devices, and agents are expected to share contextual knowledge and provide relevant services and information to users based on their situational needs [26]. A general ontology needs to be able to adapt itself, for example, to a specific $R B$ or, to a given UWS, in a $R B$ where it will be in use.

All these services need a large amount of domain-specific and general knowledge to be available, and $\mathrm{WaWO}+$ aims to be one of those sources.

1 The actual version of $W a W O+$ is obtainable at http://protegewiki.stanford.edu/wiki/Protege_Ontology_Library\#OWL_ontologies 


\section{References}

1. Agriculture meteorology sensor network. https://www.w3.org/2005/Incubator/ssn/wiki/ Agriculture_Meteorology_Sensor_Network. Accessed: 2016-02-11.

2. Consorci per a la Defensa de la Conca del Besòs. http://besos.cat/la-conca/ la-conca-del-besos/. Accessed: 2015-10-05.

3. An institutional analysis of methods for environmental appraisal. Ecological Economics, 68(8-9):2207 - 2215, 2009.

4. Agnar Aamodt and Enric Plaza. Case-based reasoning: Foundational issues, methodological variations, and system approaches. AI communications, 7(1):39-59, 1994.

5. Huib Aldewereld, Sergio Álvarez-Napagao, Frank Dignum, and Javier Vázquez-Salceda. Making norms concrete. In Proceedings of the 9th International Conference on Autonomous Agents and Multiagent Systems: volume 1-Volume 1, pages 807-814. International Foundation for Autonomous Agents and Multiagent Systems, 2010.

6. D. Allemang and J. Hendler. Semantic Web for the Working Ontologist, Effective Modeling in RDF and OWL. Morgan Kaufman, 2008.

7. Gabriel Anzaldi-Varas. Generic ontology for water supply distribution chain. Deliverable D1.3. Ref. 318603, Water Enhanced Resource Planning - Where water supply meets demand, Barcelona, Spain, December 2013.

8. Montse Aulinas, Juan Carlos Nieves, Ulises Cortés, and Manel Poch. Supporting decision making in urban wastewater systems using a knowledge-based approach. Environmental Modelling and Software, 26(5):562-572, 2011.

9. Montse Aulinas, Juan Carlos Nieves, Manel Poch, and Ulises Cortés. Supporting Decision Making in River Basin Systems Using a Declarative Reasoning Approach. In M. Finkel and P. Grathwohl, editors, Proceedings of the AquaTerra Conference (Scientific Fundamentals for River Basic Management), ISSN 0935-4948, page 75, March 2009.

10. Montse Aulinas, Pancho Tolchinsky, Clàudia Turon, Manel Poch, and Ulises Cortés. Argumentation-based framework for industrial wastewater discharges management. Eng. Appl. of AI, 25(2):317-325, 2012.

11. Franz Baader. The description logic handbook: Theory, implementation and applications. Cambridge university press, 2003.

12. T. Balke. Towards the Governance of Open Distributed Grids - A Case Study in Wireless Mobile Grids. PhD thesis, U. Bayreuth, 2011.

13. Simon Elias Bibri. The human face of Ambient Intelligence: Cognitive, emotional, affective, behavioral and conversational aspects, volume 9. Springer, 2015.

14. Guido Boella and Leendert WN van der Torre. Regulative and constitutive norms in normative multiagent systems. KR, 4:255-265, 2004.

15. Jan Broersen, Frank Dignum, Virginia Dignum, and John-Jules Ch Meyer. Designing a deontic logic of deadlines. In Deontic logic in computer science, pages 43-56. Springer, 2004.

16. Bruce G Buchanan, Edward Hance Shortliffe, et al. Rule-based expert systems, volume 3. Addison-Wesley Reading, MA, 1984

17. Violeta Cabello, Barbara A. Willaarts, Monica Aguilar, and Leandro del Moral Ituarte. River basins as social-ecological systems: linking levels of societal and ecosystem water metabolism in a semiarid watershed. Ecology and Society, 20(3):20, 2015.

18. Octavio Cabezut-Boo and Antonio Sánchez-Aguilar. Towards an ontology of waste water treatment plants: the identification phase. Environmental modelling $\& 5$ software, 14(5):401-408, 1999.

19. CEC. Directive concerning urban waste water treatment (91/271/EEC), volume 30. Official/Journal/L135/40, 1991.

20. CEC. Directive 2000/60/ec of the European Parliament and of the Council establishing a Framework for Community Action in the Field of Water Policy. Oficial Journal of the European Communities on 22 December (OJ L 327), pages 0001:0073, 2000.

21. Luigi Ceccaroni. ONTOWEDSS-An Ontology-based Environmental Decision Support System for the Management of Wastewater Treatment Plants. PhD thesis, Universitat Politècnica de Catalunya, 2001. 
22. Luigi Ceccaroni. An Environmental Decision-support System for Wastewater Treatment: Bridging Environmental Science and Information and Communication Technology. LAP Lambert Academic Publishing, Germany, 2009.

23. Luigi Ceccaroni, Ulises Cortés, and Miquel Sanchez-Marre. Ontowedss: augmenting environmental decision-support systems with ontologies. Environmental Modelling \& Software, 19(9):785-797, 2004.

24. Luigi Ceccaroni and Luis Oliva. Ontologies for the design of ecosystems. Universal Ontology of Geographic Space: Semantic Enrichment for Spatial Data: Semantic Enrichment for Spatial Data, page 207, 2012.

25. K. W. Chau. An Ontology-based knowledge management system for flow and water quality modeling. Advances in Engineering Software, 38(3):172-181, 2007.

26. Harry Chen, Tim Finin, and Anupam Joshi. An ontology for context-aware pervasive computing environment. The Knowledge Engineering Review, 18(03):197-207, 2003.

27. EFRA Committee. Water for life and livelihoods. river Basin Management Plan. Report, Environment Agency, Thames River Basin District, December 2009.

28. Generalitat de Catalunya. Psaru 2005. http://aca-web.gencat.cat/aca/appmanager/aca/ aca?_nfpb=true\&_pageLabel=P1204654461208200526170\&profileLocale=es. Accessed: 201503-30.

29. F. Devesa, J. Comas, C. Turon, A. Freixo, F. Carrasco, and M. Poch. Scenario analysis for the role of sanitation infrastructures in integrated urban wastewater management. Environmental Modelling and Software, 24(3):371-380, 2009.

30. Directive. Directive $80 / 777$ /eec of 15 july 1980 on the approximation of the laws of the member states relating to the exploitation and marketing of natural mineral waters, July 1980.

31. Directive. Directive $98 / 43 /$ ec of the european parliament and of the council of 3 november 1998 on the quality of water intended for human consumption, November 1998.

32. ENvO. Environment Ontology. http://www.environmentontology.org, 2013.

33. Adam Farquhar, Richard Fikes, and James Rice. The ontolingua server: A tool for collaborative ontology construction. International journal of human-computer studies, 46(6):707-727, 1997.

34. Mariano Fernández-López, Asunción Gómez-Pérez, and Natalia Juristo. Methontology: from ontological art towards ontological engineering. 1997.

35. Julián Garrido and Ignacio Requena. Proposal of ontology for environmental impact assessment: An application with knowledge mobilization. Expert Syst. Appl, 38(3):2462$2472,2011$.

36. Michael R Genesereth, Richard E Fikes, et al. Knowledge interchange format-version 3.0: reference manual. 1992.

37. Corentin Girard, Jean-Daniel Rinaudo, Manuel Pulido-Velazquez, and Yvan Caballero. An interdisciplinary modelling framework for selecting adaptation measures at the river basin scale in a global change scenario. Environmental Modelling 8 Software, 69:42-54, 2015.

38. N. Guarino. Formal ontology and information systems. In N. Guarino, editor, Formal Ontology in Information Systems (FOIS), pages 3-18. IOS Press, Amsterdam, 1998.

39. J. Horsburgh, D. Tarboton, M. Piasecki, D. Maidment, I. Zaslavsky, D. Valentine, and T. Whitenack. An integrated system for publishing environmental observations data. Environmental Modelling \& Software, 24:879-888, 2009.

40. Daniel S Kaster, Claudia B Medeiros, and Heloisa V Rocha. Supporting modeling and problem solving from precedent experiences: the role of workflows and case-based reasoning. Environmental Modelling \& Software, 20(6):689-704, 2005.

41. Nils Kehrein, Jürgen Berlekamp, and Jörg Klasmeier. Modeling the fate of down-thedrain chemicals in whole watersheds: New version of the great-er software. Environmental Modelling \& Software, 64:1-8, 2015.

42. Holger Knublauch, Mark A. Musen, and Alan L. Rector. Editing description logic ontologies with the protégé OWL plugin. In Volker Haarslev and Ralf Möller, editors, Proceedings of the 2004 International Workshop on Description Logics (DL2004), Whistler, British Columbia, Canada, June 6-8, 2004, volume 104 of CEUR Workshop Proceedings. CEUR-WS.org, 2004.

43. Thilo Koegst. Ontology based model framework for conceptual design of treatment flow sheets. PhD thesis, Technische Universitat Dresden, 2013. 
44. Arnim Kuhn, Wolfgang Britz, Daniel Kyalo Willy, and Pieter van Oel. Simulating the viability of water institutions under volatile rainfall conditions-the case of the lake naivasha basin. Environmental Modelling \& Software, 2014.

45. W. Medema, B. S. McIntosh, and P. J. Jeffrey. From premise to practice: a critical assessment of integrated water resources management and adaptive management approaches in the water sector. Ecology and Society, 13(2):29, 2008.

46. P Mikuleckỳ, D Ponce, et al. Knowledge-based approaches for river basin management. Hydrology and Earth System Sciences Discussions Discussions, 4(3):1999-2033, 2007.

47. E. Muñoz, E. Capón-García, José M. Laínez, A. Espuña, and L. Puigjaner. Ontological framework for the enterprise from a process perspective - operational, tactical and strategic integration for improved decision-making. In Joaquim Filipe and Jan L. G. Dietz, editors, KEOD 2011 - Proceedings of the International Conference on Knowledge Engineering and Ontology Development, Paris, France, 26-29 October, 2011, pages 538-546. SciTePress, 2011.

48. Juan Carlos Nieves, Dario Garcia-Gasulla, Montse Aulinas, and Ulises Cortés. An operational approach for implementing normative agents in urban wastewater systems. Computación y Sistemas, 16(1), 2012.

49. Natalya F Noy, Monica Crubézy, Ray W Fergerson, Holger Knublauch, Samson W $\mathrm{Tu}$, Jennifer Vendetti, Mark A Musen, et al. Protege-2000: an open-source ontologydevelopment and knowledge-acquisition environment. In AMIA Annu Symp Proc, volume 953, page 953, 2003.

50. Natalya F. Noy and Deborah L. Mcguinness. Ontology development 101: A guide to creating your first ontology. Technical report, 2001.

51. Viral Parekh. Applying Ontologies and Semantic Web technologies to Environmental Sciences and Engineering. Master thesis, University of Maryland, 2005.

52. Maria Teresa Pazienza and Michele Vindigni. Agent to agent talk: "nobody there?" supporting agents linguistic communication. In Ontologies for Agents: Theory and Experiences, pages 43-72. Springer, 2005.

53. Hardy Pundt and Yaser Bishr. Domain ontologies for data sharing - an example from environmental monitoring using field GIS. Computers \& Geosciences, 28:95-102, 2002.

54. Thania Rendón-Sallard, Miquel Sànchez-Marrè, Montserrat Aulinas, and Joaquim Comas. Designing a multi-agent system to simulate scenarios for decision-making in river basin systems. In Proc. of the 2006 Conf. on Artificial Intelligence Research and Development, pages 291-298. IOS Press, 2006.

55. Miquel Sànchez, Ulises Cortés, Javier Lafuente, Ignasi R Roda, and Manel Poch. Daidepur: an integrated and distributed architecture for wastewater treatment plants supervision. Artificial Intelligence in Engineering, 10(3):275-285, 1996.

56. John R Searle. The construction of social reality. Simon and Schuster, 1995.

57. J. A. Sheth and J.Larson. Federated database systems for managing distributed, heterogeneous, and autonomous database. ACM Computing Survey, 22:183-236, 1990.

58. Herbert A. Simon. Rational decision making in business organizations. The American Economic Review, 69(4):493-513, 1979.

59. Evren Sirin, Bijan Parsia, Bernardo Cuenca Grau, Aditya Kalyanpur, and Yarden Katz. Pellet: A practical owl-dl reasoner. Web Semantics: science, services and agents on the World Wide Web, 5(2):51-53, 2007.

60. D. Sottara, J. C. Correale, T. Spetebroot, D. Pulcini, D. Giunchi, F. Paolucci, and L. Luccarini. An ontology-based approach for the instrumentation,control and automation infrastructure of a WWTP. In Proceedings of the rth Int. Congress on Env. Modelling and Software,. iEMS, 2014.

61. Ovidiu Vermesan and Peter Friess. Internet of Things: converging technologies for smart environments and integrated ecosystems. River Publishers, 2013.

62. F. Villa, I. N. Athanasiadis, and A. E. Rizzoli. Modelling with knowledge: A review of emerging semantic approaches to environmental modelling. Environmental Modelling \& Software, 24(5):577-587, 2009.

63. Ferdinando Villa, Ioannis N Athanasiadis, and Andrea Emilio Rizzoli. Modelling with knowledge: A review of emerging semantic approaches to environmental modelling. Environmental Modelling \& Software, 24(5):577-587, 2009. 
64. Martin Volk, Jesko Hirschfeld, Alexandra Dehnhardt, Gerd Schmidt, Carsten Bohn, Stefan Liersch, and Philip W Gassman. Integrated ecological-economic modelling of water pollution abatement management options in the Upper Ems River Basin. Ecological Economics, 66(1):66-76, 2008.

65. Johanna Völker. Learning Expressive Ontologies, volume 2 of Studies on the Semantic Web. IOS Press, 2009.

66. Michael Wooldridge. An Introduction to MultiAgent Systems. Wiley Publishing, $2^{\text {nd }}$ edition, 2009.

67. I. Zaslavsky, D. Valentine, and T. Whiteaker. CUASHI WaterML. Discussion paper 07-041r1, Open Geospatial Consortium, 2007. 